\title{
A space-time continuous finite element method for 2D viscoelastic wave equation
}

\author{
Hong $\mathrm{Li}^{1}$, Zhihui Zhao' and Zhendong Luo ${ }^{2^{*}}$
}

\author{
"Correspondence: \\ zhdluo@ncepu.edu.cn \\ ${ }^{2}$ School of Mathematics and \\ Physics, North China Electric Power \\ University, No. 2, Bei Nong Road, \\ Changping District, Beijing, 102206, \\ China \\ Full list of author information is \\ available at the end of the article
}

\begin{abstract}
In this article, we establish a space-time continuous finite element (STCFE) method for viscoelastic wave equation. The existence, uniqueness, and stability of the STCFE solutions are proved, and the optimal rates of convergence of STCFE solutions are obtained without any time and space mesh size restrictions. Two numerical examples on unstructured meshes are employed to verify the efficiency and feasibility of the STCFE method and to check the correctness of theoretical conclusions.

MSC: $74510 ; 65 \mathrm{M} 15 ; 35 \mathrm{Q} 35$

Keywords: space-time continuous finite element method; viscoelastic wave equation; optimal rates of convergence; numerical examples
\end{abstract}

\section{Introduction}

In this paper, we investigate the space-time continuous finite element (STCFE) method for two-dimensional (2D) viscoelastic wave equation. For convenience, without loss of generality, we consider the following initial boundary value problem of $2 \mathrm{D}$ viscoelastic wave equation.

Problem I Find $u=u(x, y, t)$ satisfying

$$
\begin{cases}u_{t t}-\varepsilon \Delta u_{t}-\gamma \Delta u=f, & (x, y, t) \in \Omega \times[0, T], \\ u(x, y, t)=\varphi(x, y, t), & (x, y, t) \in \partial \Omega \times[0, T], \\ u(x, y, 0)=\varphi_{0}(x, y), \quad u_{t}(x, y, 0)=\varphi_{1}(x, y), & (x, y) \in \Omega,\end{cases}
$$

where $\Omega \subset \mathbf{R}^{2}$ is a bounded convex polygonal region with smooth boundary $\partial \Omega, u_{t t}=$ $\partial^{2} u / \partial t^{2}, u_{t}=\partial u / \partial t, \varepsilon$ and $v$ are positive constants, and $T$ is the final time. The source term $f(x, y, t)$, the boundary value function $\varphi(x, y, t)$, and the initial value functions $\varphi_{0}(x, y)$ and $\varphi_{1}(x, y)$ are smooth enough so that the following theoretical proofs are effective.

Equation (1.1) is known as a system of viscoelastic wave equation. It is used to describe the wave propagation phenomena of actual vibration through a viscoelastic medium (see, e.g., $[1,2])$. Though the researches of numerical solutions of viscoelastic wave equation have made a great progress (see, e.g. [3-5]), most of the existing papers either used the classical finite element (FE) methods or used finite difference (FD) schemes as discretization tools (see $[6,7])$.

(c) $2016 \mathrm{Li}$ et al. This article is distributed under the terms of the Creative Commons Attribution 4.0 International License (http://creativecommons.org/licenses/by/4.0/), which permits unrestricted use, distribution, and reproduction in any medium, provided you give appropriate credit to the original author(s) and the source, provide a link to the Creative Commons license, and indicate if changes were made. 
The STCFE method is a kind of FE technique that adopts FE to discretize the temporal and spatial variables, respectively, and provides a consistent treatment of temporal and spatial discretizations. Therefore, as long as the STCFE method employs higher degrees of polynomials about time appropriately, its numerical solutions can have higher accuracy with respect to time than those of the classical FE methods, where the time derivative is discretized by Euler backward difference with first-order accuracy, and even than the Crank-Nicolson FE solutions with second-order accuracy (see, e.g., [8-10]). In addition, the theoretical analyses of the classical FE methods will certainly change with the variations of discretization methods of time derivative, whereas the theoretical analysis of the STCFE method holds for approximate subspaces with any degrees of time polynomials, so the theoretical analysis of the STCFE method is more convenient than those of the classical FE methods. Especially, the STCFE method is very suitable for wave problems because they retain energy conservation properties of the corresponding discrete problems (see [11]). Therefore, it is considered to be one of the most effective numerical methods. It plays an important role in finding numerical solutions for time-dependent partial differential equations (TDPDEs) and forms a hot research topic. It has been widely used to find numerical solutions of various types of TDPDEs, such as parabolic equations, hyperbolic equations, nonlinear Schrödinger equation, and convection diffusion equations (see [1219]).

Aziz and Monk [15] used the STCFE method to study the heat equation. Bales and Lasiecka [16] and French and Peterson [17] also investigated the wave equation by means of the STCFE method. However, to the best of our knowledge, the STCFE method was used for solving the $2 \mathrm{D}$ viscoelastic wave equation, which is different to and far more complex than the heat equation and the wave equation. Therefore, in this study, we employ the STCFE technique to study the 2D viscoelastic wave equation. However, our theoretical analysis is different from those in [15-17]; it is more concise and easier for obtaining various error estimates with different norms, so it should be an interesting work. Especially, our estimates are obtained without any restriction conditions between temporal and spatial grid sizes, so that our method is more suitable for practical applications and is different from the existing methods (see, e.g., [12-19]). Therefore, it is a kind of improvement and development of the existing papers.

The remainder of this paper is organized as follows. In Section 2, we establish the STCFE approach approximate scheme for the 2D viscoelastic wave equation. In Section 3, the optimal rates of convergence of the STCFE solutions are derived. In Section 4, some numerical experiments are provided for illustrating the correctness of the theoretical analysis. Moreover, we verify that the STCFE method is more feasible and efficient for solving viscoelastic wave equation than the classical FE methods. Section 5 gives the main conclusions and some perspectives.

\section{STCFE method for 2D viscoelastic wave equation}

The Sobolev spaces and norms along with their theories applied in this paper are standard (see [20]). The spaces $H^{s}(\Omega)$ are equipped with the norms $\|\cdot\|_{s}$ and seminorms $|\cdot|_{s}(s \geq 0)$. If $s=0$, then the space $H^{0}(\Omega)$ is written as $L^{2}(\Omega)$ with inner product $(\cdot, \cdot)$ and norm $\|\cdot\|_{0}$. In addition, we define the energy norm on $L^{2}(\Omega) \times H^{1}(\Omega)$ by $\|(v, u)\| \|=\left\{\|v\|^{2}+\|\nabla u\|^{2}\right\}^{\frac{1}{2}}$. We also use the space $H_{0}^{1}(\Omega)=\left\{v \in H^{1}(\Omega) ;\left.v\right|_{\partial \Omega}=0\right\}$ and its dual space $H^{-1}(\Omega)$. For $g \in H^{-1}(\Omega)$, 
its norm is defined by

$$
\|g\|_{-1}=\sup _{v \in H_{0}^{1}(\Omega)} \frac{\langle g, v\rangle}{\|v\|_{1}} .
$$

Moreover, space-time Sobolev spaces are defined by

$$
H^{l}\left(0, t_{n} ; H^{m}(\Omega)\right)=\left\{v(x, t) ; \sum_{i=0}^{l} \int_{0}^{t_{n}}\left\|\frac{\mathrm{d}^{i}}{\mathrm{~d} t^{i}} v(\cdot, t)\right\|_{m}^{2} \mathrm{~d} t<\infty\right\}
$$

with norms

$$
\|v\|_{H^{l}\left(0, t_{n} ; H^{m}\right)}=\left[\sum_{i=0}^{l} \int_{0}^{t_{n}}\left\|\frac{\mathrm{d}^{i}}{\mathrm{~d} t^{i}} v(\cdot, t)\right\|_{m}^{2} \mathrm{~d} t\right]^{1 / 2} .
$$

Especially, when $l=0$ and $m=0,1$, the corresponding norms are denoted by

$$
\|v\|_{L^{2}\left(0, t_{n} ; L^{2}\right)}=\left[\int_{0}^{t_{n}}\|v(\cdot, t)\|^{2} \mathrm{~d} t\right]^{1 / 2}
$$

and

$$
\|v\|_{L^{2}\left(0, t_{n} ; H^{1}\right)}=\left[\int_{0}^{t_{n}}\|v(\cdot, t)\|_{1}^{2} \mathrm{~d} t\right]^{1 / 2} .
$$

If $t_{n}=T$, then $\|v\|_{H^{l}\left(0, t_{n} ; H^{m}\right)}$ are denoted by $\|v\|_{H^{l}\left(H^{m}\right)}$.

We reformulate Problem I as a first-order system with respect to time by introducing the function $v=u_{t}$. Thus, Problem I may be rewritten as follows.

Problem II Find $(u, v)$ such that

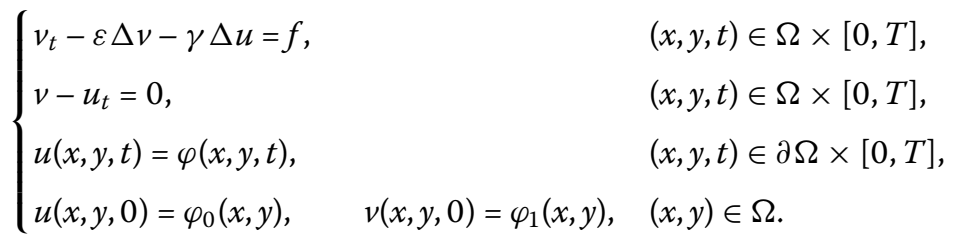

For convenience and without loss of generality, we may also suppose that $\varphi(x, y, t)$, $\varphi_{0}(x, y)$, and $\varphi_{1}(x, y)$ are all zero functions in the following theoretical analysis. Let $U=$ $H^{1}\left(0, T ; H_{0}^{1}(\Omega)\right)$. Thus, we can write the weak formulation for Problem II as follows.

Problem III Find $(u, v) \in U \times U$ such that

$$
\begin{aligned}
& \int_{0}^{T}\left[\left(v, w_{t}\right)-\left(u_{t}, w_{t}\right)\right] \mathrm{d} t=0, \quad \forall w \in U, \\
& \int_{0}^{T}\left[\left(v_{t}, z_{t}\right)+\varepsilon a\left(v, z_{t}\right)+\gamma a\left(u, z_{t}\right)\right] \mathrm{d} t=\int_{0}^{T}\left(f, z_{t}\right) \mathrm{d} t, \quad \forall z \in U, \\
& u(x, y, 0)=0, \quad v(x, y, 0)=0, \quad(x, y) \in \Omega,
\end{aligned}
$$

where $a(u, v)=(\nabla u, \nabla v)$. 
In order to construct the SCTFE formulation, let $\mathfrak{\Im}_{h}=\{K\}$ be a quasi-uniform triangulation subdivision of computational region $\bar{\Omega}$ with $h=\max h_{K}$, where $h_{K}$ denotes the diameter of the triangle $K \in \Im_{h}$ (see $[8,21,22]$ ), and take a partition $0=t_{0}<t_{1}<\cdots<t_{N}=T$ on time span $[0, T]$ with the time step $k=\max _{1 \leq j \leq N}\left|t_{j}-t_{j-1}\right|$. Then, we introduce the subspace $S_{h m}(\Omega) \subset H_{0}^{1}(\Omega)$ consisting of piecewise continuous polynomials of degree $m$ defined on the partition $\Im_{h}$ of $\Omega$ with mesh parameter $h$. Let $S_{k l}([0, T])$ be a finite element subspace on time partition consisting of piecewise continuous polynomials of degree $l$, that is, $S_{k l}([0, T])=\left\{v \in C^{0}([0, T]):\left.v\right|_{\left[t_{j-1}, t_{j}\right]} \in P_{l}\left(\left[t_{j-1}, t_{j}\right]\right), j=1, \ldots, N\right\}$, where $P_{l}\left(\left[t_{j-1}, t_{j}\right]\right)$ is the set of polynomials $0=t_{0}<t_{1}<\cdots<t_{N}=T$ of degrees not higher than $l$. Finally, we define space-time element subspace $U_{h k}=S_{h m}(\Omega) \otimes S_{k l}([0, T])$. Then, the STCFE formulation for $2 \mathrm{D}$ wave equations is established as follows.

Problem IV Find $\left(u^{h k}, v^{h k}\right) \in U_{h k}^{2}$ such that

$$
\begin{aligned}
& \int_{0}^{T}\left[\left(v^{h k}, w_{t}\right)-\left(u_{t}^{h k}, w_{t}\right)\right] \mathrm{d} t=0, \quad \forall w \in U_{h k}, \\
& \int_{0}^{T}\left[\left(v_{t}^{h k}, z_{t}\right)+\varepsilon a\left(v^{h k}, z_{t}\right)+\gamma a\left(u^{h k}, z_{t}\right)\right] \mathrm{d} t=\int_{0}^{T}\left(f, z_{t}\right) \mathrm{d} t, \quad \forall z \in U_{h k}, \\
& u^{h k}(x, y, 0)=0, \quad v^{h k}(x, y, 0)=0, \quad(x, y) \in \Omega .
\end{aligned}
$$

The STCFE solution pair $\left(u^{h k}, v^{h k}\right)$ can be found by advancing via successive time levels. To this end, let $J_{n}=\left[t_{n-1}, t_{n}\right]$, and let $P_{l}\left(J_{n}\right)$ be the set of polynomial functions on the time interval $J_{n}$ of degree not higher than $l$. Then, for $n=1,2, \ldots, N$, the STCFE solution pair $\left(u^{h k}, v^{h k}\right)$ is found as the unique solution of

$$
\begin{aligned}
& \int_{J_{n}}\left[\left(v^{h k}, w_{t}\right)-\left(u_{t}^{h k}, w_{t}\right)\right] \mathrm{d} t=0, \quad \forall w \in S_{h m}(\Omega) \otimes P_{l}\left(J_{n}\right), \\
& \int_{J_{n}}\left[\left(v_{t}^{h k}, z_{t}\right)+\varepsilon a\left(v^{h k}, z_{t}\right)+\gamma a\left(u^{h k}, z_{t}\right)\right] \mathrm{d} t=\int_{J_{n}}\left(f, z_{t}\right) \mathrm{d} t, \quad \forall z \in S_{h m}(\Omega) \otimes P_{l}\left(J_{n}\right),
\end{aligned}
$$

or is equivalently written as

$$
\begin{aligned}
& \int_{J_{n}}\left[\left(v^{h k}, w\right)-\left(u_{t}^{h k}, w\right)\right] \mathrm{d} t=0, \quad \forall w \in S_{h m}(\Omega) \otimes P_{l-1}\left(J_{n}\right), \\
& \int_{J_{n}}\left[\left(v_{t}^{h k}, z\right)+\varepsilon a\left(v^{h k}, z\right)+\gamma a\left(u^{h k}, z\right)\right] \mathrm{d} t \\
& \quad=\int_{J_{n}}(f, z) \mathrm{d} t, \quad \forall z \in S_{h m}(\Omega) \otimes P_{l-1}\left(J_{n}\right),
\end{aligned}
$$

with $u^{h k}(x, y, 0)=0, v^{h k}(x, y, 0)=0$, where $u^{h k}\left(x, y, t_{n}\right), v^{h k}\left(x, y, t_{n}\right)(n=1,2, \ldots, N-1,(x, y) \in$ $\Omega$ ) are given and have been found at the previous time step.

Remark 1 The system of equations (2.10)-(2.11) can be seen by applying Petrov-Galerkin approach to approximate the viscoelastic wave equation, where the trial functions $v^{h k}$ and $u^{h k}$ are continuous with respect to time and space, whereas the test functions $w$ and $z$ are space-continuous and time-discontinuous. 
In order to discuss the existence, uniqueness, and stability for Problem IV, it is necessary to introduce the discrete operator $A_{h}: L^{2}\left(0, T ; H_{0}^{1}(\Omega)\right) \rightarrow S_{h m}(\Omega) \times L^{2}(0, T)$ defined by

$$
\int_{0}^{T}\left(A_{h} u, \phi\right) \mathrm{d} t=\int_{0}^{T}(\nabla u, \nabla \phi) \mathrm{d} t, \quad \forall \phi \in S_{h m}(\Omega) \otimes L^{2}(0, T) .
$$

Theorem 1 If $\in L^{2}\left(0, t_{n} ; L^{2}(\Omega)\right)$, then there exists a unique solution pair $\left(u^{h k}, v^{h k}\right) \in U_{h k}^{2}$ to Problem IV such that

$$
\begin{gathered}
\left\|v^{h k}\left(t_{n}\right)\right\|_{0}+\left\|\nabla u_{t}^{h k}\right\|_{L^{2}\left(0, t_{n} ; L^{2}(\Omega)\right)}+\left\|\nabla u^{h k}\left(t_{n}\right)\right\|_{0} \\
\leq C\|f\|_{L^{2}\left(0, t_{n} ; L^{2}(\Omega)\right)}, \quad n=1,2, \ldots, N
\end{gathered}
$$

where $C$ is a positive constant depending on $\varepsilon$ and $\gamma$ but is always independent of $h$ and $k$ and may be different at different places.

Proof Because Problem IV is a linear system of equations, in order to prove the existence and uniqueness of the solution pair for Problem IV, it is necessary to demonstrate that if $f=0$, then there exists a unique zero solution pair to Problem IV.

Taking $w=v_{t}^{h k}$ in (2.10) and $z=u_{t}^{h k}$ in (2.11) yields

$$
\int_{J_{n}}\left[\left(v^{h k}, v_{t}^{h k}\right)+\varepsilon a\left(v^{h k}, u_{t}^{h k}\right)+\gamma a\left(u^{h k}, u_{t}^{h k}\right)\right] \mathrm{d} t=\int_{J_{n}}\left(f, u_{t}^{h k}\right) \mathrm{d} t .
$$

Further, taking $w=A_{h} u_{t}^{h k}$ in (2.10) and combining (2.12) with (2.14), we have

$$
\int_{J_{n}}\left[\left(v^{h k}, v_{t}^{h k}\right)+\varepsilon a\left(u_{t}^{h k}, u_{t}^{h k}\right)+\gamma a\left(u^{h k}, u_{t}^{h k}\right)\right] \mathrm{d} t=\int_{J_{n}}\left(f, u_{t}^{h k}\right) \mathrm{d} t
$$

Equation (2.15) is equivalently written as follows:

$$
\int_{J_{n}}\left[\frac{1}{2} \frac{\mathrm{d}}{\mathrm{d} t}\left\|v^{h k}\right\|_{0}^{2}+\varepsilon\left\|\nabla u_{t}^{h k}\right\|_{0}^{2}+\frac{\gamma}{2} \frac{\mathrm{d}}{\mathrm{d} t}\left\|\nabla u^{h k}\right\|_{0}^{2}\right] \mathrm{d} t=\int_{J_{n}}\left(f, u_{t}^{h k}\right) \mathrm{d} t .
$$

Noting that $\|u\|_{0} \leq c\|\nabla u\|_{0}$ in $H_{0}^{1}(\Omega)$ (where $\mathrm{c}$ is a positive constant independent of $h$ and $k$, possibly different at different occurrences). By the Hölder and Cauchy inequalities, for the right-hand side of (2.16), we have

$$
\begin{aligned}
\int_{J_{n}} & {\left[\frac{1}{2} \frac{\mathrm{d}}{\mathrm{d} t}\left\|v^{h k}\right\|_{0}^{2}+\varepsilon\left\|\nabla u_{t}^{h k}\right\|_{0}^{2}+\frac{\gamma}{2} \frac{\mathrm{d}}{\mathrm{d} t}\left\|\nabla u^{h k}\right\|_{0}^{2}\right] \mathrm{d} t } \\
& \leq \int_{J_{n}}\|f\|_{0}\left\|u_{t}^{h k}\right\|_{0} \mathrm{~d} t \leq \int_{J_{n}} c\|f\|_{0}\left\|\nabla u_{t}^{h k}\right\|_{0} \mathrm{~d} t \\
& \leq \int_{J_{n}} \frac{1}{2}\left[\frac{c^{2}}{\varepsilon}\|f\|_{0}^{2}+\varepsilon\left\|\nabla u_{t}^{h k}\right\|_{0}^{2}\right] \mathrm{d} t .
\end{aligned}
$$

Thus, inequality (2.17) can be simplified as

$$
\int_{J_{n}}\left[\frac{\mathrm{d}}{\mathrm{d} t}\left\|v^{h k}\right\|_{0}^{2}+\varepsilon\left\|\nabla u_{t}^{h k}\right\|_{0}^{2}+\gamma \frac{\mathrm{d}}{\mathrm{d} t}\left\|\nabla u^{h k}\right\|_{0}^{2}\right] \mathrm{d} t \leq \frac{c^{2}}{\varepsilon} \int_{J_{n}}\|f\|_{0}^{2} \mathrm{~d} t .
$$


Further, we obtain

$$
\begin{aligned}
& \left\|v^{h k}\left(t_{n}\right)\right\|_{0}^{2}+\varepsilon\left\|\nabla u_{t}^{h k}\right\|_{L^{2}\left(J_{n} ; L^{2}\right)}^{2}+\gamma\left\|\nabla u^{h k}\left(t_{n}\right)\right\|_{0}^{2} \\
& \quad \leq \frac{c^{2}}{\varepsilon}\|f\|_{L^{2}\left(J_{n} ; L^{2}\right)}^{2}+\left\|v^{h k}\left(t_{n-1}\right)\right\|_{0}^{2}+\gamma\left\|\nabla u^{h k}\left(t_{n-1}\right)\right\|_{0}^{2}, \quad n=1,2, \ldots, N .
\end{aligned}
$$

Since $u^{h k}(x, y, 0)=0$ and $v^{h k}(x, y, 0)=0$, by summing (2.19) from 1 to $n$ we obtain

$$
\begin{gathered}
\left\|v^{h k}\left(t_{n}\right)\right\|_{0}^{2}+\varepsilon\left\|\nabla u_{t}^{h k}\right\|_{L^{2}\left(0, t_{n} ; L^{2}\right)}^{2}+\gamma\left\|\nabla u^{h k}\left(t_{n}\right)\right\|_{0}^{2} \\
\leq \frac{c^{2}}{\varepsilon}\|f\|_{L^{2}\left(0, t_{n} ; L^{2}\right)}^{2}, \quad n=1,2, \ldots, N .
\end{gathered}
$$

If $f=0$, then from (2.20) we get that $u_{t}^{h k}(x, y, t)=v^{h k}\left(t_{n}\right)=\nabla u^{h k}\left(t_{n}\right)=0\left((x, y, t) \in \Omega \times J_{n}\right.$, $n=1,2, \ldots, N)$. Further, from $u^{h k}(x, y, 0)=0$ we obtain $u^{h k}(x, y, t)=0((x, y, t) \in \Omega \times[0, T])$. In addition, taking $z=v_{t}^{h k}$ in (2.11) and then summing from $n=1$ to $N$, we get $\left\|v_{t}^{h k}\right\|_{L^{2}\left(L^{2}\right)}=$ $\left\|\nabla v^{h k}(T)\right\|_{0}=0((x, y, t) \in \Omega \times[0, T])$, which implies that $v^{h k}(x, y, t)=0((x, y, t) \in \Omega \times$ $[0, T])$ since $v^{h k}(x, y, 0)=v^{h k}\left(x, y, t_{n}\right)=0\left((x, y, t) \in \Omega \times J_{n}, n=1,2, \ldots, N\right)$. Therefore, Problem IV has a unique solution pair $\left(u^{h k}, v^{h k}\right) \in U_{h k}^{2}$. From (2.20) we immediately obtain (2.13), which finishes the proof of Theorem 1.

\section{Error estimates of the TSCFE solutions}

To estimate the errors between exact and STCFE solutions, we need to define a spacevariable Ritz projection $P_{h}: H_{0}^{1}(\Omega) \rightarrow S_{h m}(\Omega)$; namely, for $u \in H_{0}^{1}(\Omega)$, we have

$$
\left(\nabla P_{h} u, \nabla \phi_{h}\right)=\left(\nabla u, \nabla \phi_{h}\right), \quad \forall \phi_{h} \in S_{h m}(\Omega)
$$

Owing to the regularity of the triangulation $\mathfrak{\Im}_{h}$, it is well known (see $\left.[8,21]\right)$ that $P_{h}$ has the following approximation properties. If $u \in H_{0}^{1}(\Omega) \cap H^{r}(\Omega)$, then

$$
\left\|P_{h} u-u\right\|_{s} \leq c h^{r-s}\|u\|_{r}, \quad 1 \leq r \leq m+1, s=0,1 .
$$

The projection $P_{h}$ can be extended to functions of $x, y$ and $\mathrm{t}$ in an $L^{2}$ sense. Thus, we define the extended projection $P_{h}: H^{1}\left(0, T ; H_{0}^{1}(\Omega)\right) \rightarrow S_{h m}(\Omega) \times L^{2}(0, T)$ by

$$
\int_{0}^{T}\left(\nabla P_{h} u, \nabla \phi\right) \mathrm{d} t=\int_{0}^{T}(\nabla u, \nabla \phi) \mathrm{d} t, \quad \forall \phi \in S_{h m}(\Omega) \times L^{2}(0, T) .
$$

Next, we define the solution operator $T: H^{-1}(\Omega) \rightarrow H_{0}^{1}(\Omega)$ of the Dirichlet problem for the Laplace equation on $\Omega$ and its FE approximate operator $T_{h}: H^{-1}(\Omega) \rightarrow S_{h m}(\Omega)$ as follows. For $g \in H^{-1}(\Omega)$, there exist $T g \in H_{0}^{1}(\Omega)$ and $T_{h} g \in S_{h m}(\Omega)$ such that

$$
\begin{aligned}
& (\nabla T g, \nabla \phi)=(g, \phi), \quad \forall \phi \in H_{0}^{1}(\Omega), \\
& \left(\nabla T_{h} g, \nabla \phi_{h}\right)=\left(g, \phi_{h}\right), \quad \forall \phi_{h} \in S_{h m}(\Omega) .
\end{aligned}
$$

From (3.5) we know that $T_{h}$ is a symmetric and positive operator. Further, $T_{h}$ satisfies the following bound (see [12]):

$$
0 \leq\left(g, T_{h} g\right) \leq c\|g\|_{-1}^{2}, \quad \forall g \in H^{-1}(\Omega) .
$$


Finally, we also need to define the time projection $P_{k}: H^{1}(0, T) \rightarrow S_{k l}([0, T])$; namely, for $w \in H^{1}(0, T)$, we have

$$
\int_{0}^{T}\left(P_{k} w\right)_{t} \phi_{t}^{k} \mathrm{~d} t=\int_{0}^{T} w_{t} \phi_{t}^{k} \mathrm{~d} t, \quad \forall \phi^{k} \in S_{k l}([0, T])
$$

By standard FE techniques we can easily derive that $P_{k}$ satisfies the following estimate: for $w \in H^{1}(0, T) \cap H^{r}(0, T)$,

$$
\left\|P_{k} w-w\right\|_{H^{s}(0, T)} \leq c h^{r-s}\|w\|_{H^{r}(0, T)}, \quad-l+1 \leq s \leq 1 \leq r \leq l+1 .
$$

Also, we can extend $P_{k}$ to functions of $x, y$, and $t$ in an $L^{2}$ sense. Thus, we define the extended time projection $P_{k}: H^{1}\left(0, T ; L^{2}(\Omega)\right) \rightarrow L^{2}(\Omega) \times S_{k l}([0, T])$ by

$$
\int_{0}^{T}\left(\left(P_{k} w\right)_{t}, \phi_{t}^{k}\right) \mathrm{d} t=\int_{0}^{T}\left(w_{t}, \phi_{t}^{k}\right) \mathrm{d} t, \quad \forall \phi^{k} \in L^{2}(\Omega) \otimes S_{k l}([0, T])
$$

with the initial condition $\left(P_{k} w(0), \phi\right)=(w(0), \phi)\left(\forall \phi \in L^{2}(\Omega)\right)$. Further, we take $P_{k} w\left(t_{n}\right)=$ $w\left(t_{n}\right)(n=0,1,2, \ldots, N)$. In addition, we have the following properties (see [12]).

Lemma 1 If $v \in H^{2}\left(0, T ; H^{2}(\Omega)\right)$, then

$$
\left(P_{h} v\right)_{t}=P_{h} v_{t}, \quad \nabla\left(P_{k} v\right)=P_{k} \nabla v, \quad P_{h} P_{k} v=P_{k} P_{h} v, \quad T_{h} P_{k} v=P_{k} T_{h} v
$$

Let $P_{h}$ and $P_{k}$ be defined in the extended sense by (3.3) and (3.9).

(1) If $v \in H^{r}\left(0, t_{n} ; L^{2}(\Omega)\right)$, then, for $-l+1 \leq s \leq 1 \leq r \leq l+1$, we have

$$
\int_{\Omega} \sum_{m=1}^{n}\left\|v-P_{k} v\right\|_{\left.H^{s} J_{n}\right)}^{2} \mathrm{~d} x \mathrm{~d} y \leq c k^{2(r-s)}\|v\|_{H^{r}\left(0, t_{n} ; L^{2}(\Omega)\right)}^{2} .
$$

(2) If $v \in H^{1}\left(0, T ; H^{m+1}(\Omega)\right) \cap H^{1}\left(0, T ; H_{0}^{1}(\Omega)\right)$, then

$$
\left\|\left(v-P_{h} v\right)(t)\right\|_{s} \leq c h^{m+1-s}\|v(t)\|_{m+1}, \quad s=0,1
$$

(3) If $v \in L^{2}\left(0, t_{n} ; H^{r}(\Omega)\right) \cap H^{1}\left(0, t_{n} ; H_{0}^{1}(\Omega)\right)$, then

$$
\left\|\left(v-P_{h} v\right)(t)\right\|_{L^{2}\left(0, t_{n} ; L^{2}(\Omega)\right)} \leq c h^{r}\|v(t)\|_{L^{2}\left(0, t_{n} ; H^{r}(\Omega)\right)}, \quad 1 \leq r \leq m+1 .
$$

(4) If $v \in H^{l+1}\left(0, t_{n} ; L^{2}(\Omega)\right) \cap H^{1}\left(0, t_{n} ; H_{0}^{1}(\Omega)\right)$ and $v_{t} \in L^{2}\left(0, t_{n} ; H^{m+1}(\Omega)\right) \cap H^{1}\left(0, t_{n}\right.$; $\left.H_{0}^{1}(\Omega)\right)$, then

$$
\begin{aligned}
& \left\|\left(v-P_{h} P_{k} v\right)_{t}\right\|_{L^{2}\left(0, t_{n} ; L^{2}(\Omega)\right)} \\
& \quad \leq c\left\{h^{m+1}\left\|v_{t}\right\|_{L^{2}\left(0, t_{n} ; H^{m+1}(\Omega)\right)}+k^{l}\|v\|_{H^{l+1}\left(0, t_{n} ; L^{2}(\Omega)\right)}\right\} .
\end{aligned}
$$


Lemma 2 Let $P_{h}$ and $P_{k}$ be the projections defined before, and let $u, v \in H^{1}\left(0, t_{n} ; H_{0}^{1}(\Omega)\right)$. Then, for any $(\phi, \varphi) \in U_{h k}^{2}$, we have

$$
\begin{aligned}
& \int_{0}^{t_{n}}\left[\left(\left(P_{h} v-v^{h k}\right)_{t}, \phi_{t}\right)+\varepsilon\left(\nabla\left(P_{h} v-v^{h k}\right), \nabla \phi_{t}\right)+\gamma\left(\nabla\left(P_{k} P_{h} u-u^{h k}\right), \nabla \phi_{t}\right)\right] \mathrm{d} t \\
& \quad=\int_{0}^{t_{n}}\left[\left(\left(P_{h} v-v\right)_{t}, \phi_{t}\right)+\gamma\left(\nabla\left(P_{k} u-u\right), \nabla \phi_{t}\right)\right] \mathrm{d} t
\end{aligned}
$$

and

$$
\int_{0}^{t_{n}}\left[\left(P_{h} v-v^{h k}, \varphi_{t}\right)-\left(\left(P_{k} P_{h} u-u^{h k}\right)_{t}, \varphi_{t}\right)\right] \mathrm{d} t=0
$$

Proof By the definitions of $P_{h}$ and $P_{k}$ and the properties of projections we have

$$
\begin{aligned}
\int_{0}^{t_{n}} & {\left[\left(\left(P_{h} v-v^{h k}\right)_{t}, \phi_{t}\right)+\varepsilon\left(\nabla\left(P_{h} v-v^{h k}\right), \nabla \phi_{t}\right)+\gamma\left(\nabla\left(P_{k} P_{h} u-u^{h k}\right), \nabla \phi_{t}\right)\right] \mathrm{d} t } \\
= & \int_{0}^{t_{n}}\left[\left(\left(P_{h} v-v\right)_{t}, \phi_{t}\right)+\varepsilon\left(\nabla\left(P_{h} v-v\right), \nabla \phi_{t}\right)+\gamma\left(\nabla\left(P_{k} P_{h} u-P_{h} u\right), \nabla \phi_{t}\right)\right] \mathrm{d} t \\
& +\int_{0}^{t_{n}}\left[\left(\left(v-v^{h k}\right)_{t}, \phi_{t}\right)+\varepsilon\left(\nabla\left(v-v^{h k}\right), \nabla \phi_{t}\right)+\gamma\left(\nabla\left(P_{h} u-u^{h k}\right), \nabla \phi_{t}\right)\right] \mathrm{d} t \\
= & \int_{0}^{t_{n}}\left[\left(\left(P_{h} v-v\right)_{t}, \phi_{t}\right)+\gamma\left(\nabla\left(P_{k} u-u\right), \nabla \phi_{t}\right)\right] \mathrm{d} t \\
& +\int_{0}^{t_{n}}\left[\left(\left(v-v^{h k}\right)_{t}, \phi_{t}\right)+\varepsilon\left(\nabla\left(v-v^{h k}\right), \nabla \phi_{t}\right)+\gamma\left(\nabla\left(u-u^{h k}\right), \nabla \phi_{t}\right)\right] \mathrm{d} t .
\end{aligned}
$$

In addition,

$$
\begin{gathered}
\int_{0}^{t_{n}}\left[\left(\left(P_{h} v-v^{h k}\right), \varphi_{t}\right)-\left(\left(P_{k} P_{h} u-u^{h k}\right)_{t}, \varphi_{t}\right)\right] \mathrm{d} t \\
=\int_{0}^{t_{n}}\left[\left(P_{h} v-v, \varphi_{t}\right)-\left(\left(P_{k} P_{h} u-u\right)_{t}, \varphi_{t}\right)\right] \mathrm{d} t \\
\quad+\int_{0}^{t_{n}}\left[\left(v-v^{h k}, \varphi_{t}\right)-\left(\left(u-u^{h k}\right)_{t}, \varphi_{t}\right)\right] \mathrm{d} t \\
=\int_{0}^{t_{n}}\left[\left(P_{h} v-v, \varphi_{t}\right)-\left(\left(P_{h} u-u\right)_{t}, \varphi_{t}\right)\right] \mathrm{d} t \\
\quad+\int_{0}^{t_{n}}\left[\left(v-v^{h k}, \varphi_{t}\right)-\left(\left(u-u^{h k}\right)_{t}, \varphi_{t}\right)\right] \mathrm{d} t
\end{gathered}
$$

Since $u, v$ and $u^{h k}, v^{h k}$ are solutions of Problem III and problem IV, respectively, noting that $v=u_{t}$ together with (3.17) and (3.18) finishes the proof of Lemma 2.

We state the following results on the convergence of the solutions of the equation system (2.5)-(2.7), that is, of Problem IV.

Theorem 2 Let $u(x, y, t), v(x, y, t)$ and $u^{h k}(x, y, t), v^{h k}(x, y, t)$ be the solutions of Problem III and Problem IV, respectively. Then we have the following error estimates: 
(1) Let $u(x, y, t) \in H^{m+1}(\Omega)(0 \leq t \leq T), \nabla u \in H^{l+1}\left(0, T ; L^{2}(\Omega)\right)$, and $v_{t} \in L^{2}(0, T$; $\left.H^{m+1}(\Omega)\right)$. Then

$$
\begin{aligned}
& \left\|u\left(t_{n}\right)-u^{h k}\left(t_{n}\right)\right\|_{1} \\
& \leq C\left[k^{l+1}\|\nabla u\|_{H^{l+1}\left(0, t_{n} ; L^{2}(\Omega)\right)}\right. \\
& \left.\quad+h^{m}\left(\left\|v_{t}\right\|_{L^{2}\left(0, t_{n} ; H^{m+1}(\Omega)\right)}+\left\|u\left(t_{n}\right)\right\|_{m+1}\right)\right], \quad n=1,2, \ldots, N ;
\end{aligned}
$$

(2) Let $\nabla u \in H^{l+1}\left(0, t_{n} ; L^{2}(\Omega)\right)$, $v_{t} \in L^{2}\left(0, T ; H^{m+1}(\Omega)\right)$, and $v(x, y, t) \in H^{m+1}(\Omega)$ $(0 \leq t \leq T)$. Then

$$
\begin{aligned}
\left\|\left(v\left(t_{n}\right)-v^{h k}\left(t_{n}\right)\right)\right\|_{0} \leq & C\left\{h^{m+1}\left(\left\|v\left(t_{n}\right)\right\|_{m+1}+\left\|v_{t}\right\|_{L^{2}\left(0, t_{n} ; H^{m+1}(\Omega)\right)}\right)\right. \\
& \left.+k^{l+1}\|\nabla u\|_{H^{l+1}\left(0, t_{n} ; L^{2}(\Omega)\right)}\right\}, \quad n=1,2, \ldots, N .
\end{aligned}
$$

Proof By taking $(\phi, \varphi)=\left(P_{k} P_{h} u-u^{h k}, P_{h} v-v^{h k}\right)$ in (3.15) and (3.16) we obtain

$$
\begin{aligned}
& \int_{0}^{t_{h}}\left[\left(P_{h} v-v^{h k},\left(P_{h} v-v^{h k}\right)_{t}\right)+\varepsilon\left(\nabla\left(P_{h} v-v^{h k}\right), \nabla\left(P_{k} P_{h} u-u^{h k}\right)_{t}\right)\right. \\
& \left.\quad+\gamma\left(\nabla\left(P_{k} P_{h} u-u^{h k}\right), \nabla\left(P_{k} P_{h} u-u^{h k}\right)_{t}\right)\right] \mathrm{d} t \\
& =\int_{0}^{t_{h}}\left[\left(\left(P_{h} v-v\right)_{t},\left(P_{k} P_{h} u-u^{h k}\right)_{t}\right)+\gamma\left(\nabla\left(P_{k} u-u\right), \nabla\left(P_{k} P_{h} u-u^{h k}\right)_{t}\right)\right] \mathrm{d} t .
\end{aligned}
$$

Further, setting $\varphi=A_{h}\left(P_{k} P_{h} u-u^{h k}\right)$ in (3.16), from (3.21) and (2.12) it follows that

$$
\begin{aligned}
& \int_{0}^{t_{n}}\left[\left(P_{h} v-v^{h k},\left(P_{h} v-v^{h k}\right)_{t}\right)+\varepsilon\left(\nabla\left(P_{k} P_{h} u-u^{h k}\right)_{t}, \nabla\left(P_{k} P_{h} u-u^{h k}\right)_{t}\right)\right. \\
& \left.\quad+\gamma\left(\nabla\left(P_{k} P_{h} u-u^{h k}\right), \nabla\left(P_{k} P_{h} u-u^{h k}\right)_{t}\right)\right] \mathrm{d} t \\
& =\int_{0}^{t_{n}}\left[\left(\left(P_{h} v-v\right)_{t},\left(P_{k} P_{h} u-u^{h k}\right)_{t}\right)+\gamma\left(\nabla\left(P_{k} u-u\right), \nabla\left(P_{k} P_{h} u-u^{h k}\right)_{t}\right)\right] \mathrm{d} t .
\end{aligned}
$$

Applying the Hölder and Cauchy inequalities to the right-hand side of (3.22), we have

$$
\begin{aligned}
\frac{1}{2} \| & P_{h} v\left(t_{n}\right)-v^{h k}\left(t_{n}\right)\left\|_{0}^{2}-\frac{1}{2}\right\|\left(P_{h} v(0)-v^{h k}(0)\right)\left\|_{0}^{2}+\varepsilon\right\| \nabla\left(P_{h} P_{k} u-u^{h k}\right)_{t} \|_{L^{2}\left(0, t_{n} ; L^{2}(\Omega)\right)}^{2} \\
+ & \frac{\gamma}{2}\left\|\nabla\left(P_{h} P_{k} u\left(t_{n}\right)-u^{h k}\left(t_{n}\right)\right)\right\|_{0}^{2}-\frac{\gamma}{2}\left\|\nabla\left(P_{k} P_{h} u(0)-u^{h k}(0)\right)\right\|_{0}^{2} \\
\leq & \frac{c^{2}}{\varepsilon}\left\|\left(P_{h} v-v\right)_{t}\right\|_{L^{2}\left(0, t_{n} ; L^{2}(\Omega)\right)}^{2}+\frac{\varepsilon}{4}\left\|\nabla\left(P_{h} P_{k} u-u^{h k}\right)_{t}\right\|_{L^{2}\left(0, t_{n} ; L^{2}(\Omega)\right)}^{2} \\
+ & \frac{\gamma^{2}}{\varepsilon}\left\|\nabla\left(P_{k} u-u\right)\right\|_{L^{2}\left(0, t_{n} ; L^{2}(\Omega)\right)}^{2}+\frac{\varepsilon}{4}\left\|\nabla\left(P_{h} P_{k} u-u^{h k}\right)_{t}\right\|_{L^{2}\left(0, t_{n} ; L^{2}(\Omega)\right)}^{2} .
\end{aligned}
$$

Noting that $u(0)=v(0)=0, u^{h k}(0)=v^{h k}(0)=0$, and $P_{k} P_{h} u\left(t_{n}\right)=P_{h} u\left(t_{n}\right)$, (3.23) can be simplified as follows:

$$
\begin{aligned}
& \left\|P_{h} v\left(t_{n}\right)-v^{h k}\left(t_{n}\right)\right\|_{0}+\varepsilon\left\|\nabla\left(P_{h} P_{k} u-u^{h k}\right)_{t}\right\|_{L^{2}\left(0, t_{n} ; L^{2}(\Omega)\right)}+\gamma\left\|\nabla\left(P_{h} u\left(t_{n}\right)-u^{h k}\left(t_{n}\right)\right)\right\|_{0} \\
& \leq \frac{2 c^{2}}{\varepsilon}\left\|\left(P_{h} v-v\right)_{t}\right\|_{L^{2}\left(0, t_{n} ; L^{2}(\Omega)\right)}+\frac{2 \gamma^{2}}{\varepsilon}\left\|\nabla\left(P_{k} u-u\right)\right\|_{L^{2}\left(0, t_{n} ; L^{2}(\Omega)\right)}^{2}
\end{aligned}
$$


By applying triangle inequality to (3.24) we obtain

$$
\begin{aligned}
\left\|v\left(t_{n}\right)-v^{h k}\left(t_{n}\right)\right\|_{0} \leq & \left\|v\left(t_{n}\right)-P_{h} v\left(t_{n}\right)\right\|_{0}+\left\|P_{h} v\left(t_{n}\right)-v^{h k}\left(t_{n}\right)\right\|_{0} \\
\leq & \left\|v\left(t_{n}\right)-P_{h} v\left(t_{n}\right)\right\|_{0}+\frac{2 c^{2}}{\varepsilon}\left\|\left(P_{h} v-v\right)_{t}\right\|_{L^{2}\left(0, t_{n} ; L^{2}(\Omega)\right)} \\
& +\frac{2 \gamma^{2}}{\varepsilon}\left\|\nabla\left(P_{k} u-u\right)\right\|_{L^{2}\left(0, t_{n} ; L^{2}(\Omega)\right)} .
\end{aligned}
$$

In view of (3.25), using the approximation properties of $P_{h}$ and $P_{k}$ in Lemma 1, we get (3.20). In the same way, using the triangle inequality and (3.24), we may write

$$
\begin{aligned}
& \left\|\nabla\left(u\left(t_{n}\right)-u^{h k}\left(t_{n}\right)\right)\right\|_{0} \\
& \leq\left\|\nabla\left(u\left(t_{n}\right)-P_{k} P_{h} u\left(t_{n}\right)\right)\right\|_{0}+\left\|\nabla\left(P_{k} P_{h} u\left(t_{n}\right)-u^{h k}\left(t_{n}\right)\right)\right\|_{0} \\
& \leq\left\|\nabla\left(u\left(t_{n}\right)-P_{k} P_{h} u\left(t_{n}\right)\right)\right\|_{0}+\frac{2 c^{2}}{\varepsilon \gamma}\left\|\left(P_{h} v-v\right)_{t}\right\|_{L^{2}\left(0, t_{n} ; L^{2}(\Omega)\right)} \\
& \quad+\frac{2 \gamma}{\varepsilon}\left\|\nabla\left(P_{k} u-u\right)\right\|_{L^{2}\left(0, t_{n} ; L^{2}(\Omega)\right)^{*}}
\end{aligned}
$$

Thus, (3.19) directly follows from (3.26) and Lemma 1.

In the following corollary, we provide the energy norm estimate.

Corollary 1 Under the assumptions of Theorem 2, we have the following estimate:

$$
\begin{aligned}
\| v\left(t_{n}\right) & -v^{h k}\left(t_{n}\right)\left\|_{0}+\right\| \nabla\left(u\left(t_{n}\right)-u^{h k}\left(t_{n}\right)\right) \|_{0} \\
\leq & C\left[k^{l+1}\|\nabla u\|_{H^{l+1}\left(0, t_{n} ; L^{2}(\Omega)\right)}\right. \\
& \left.+h^{m}\left(\left\|v_{t}\right\|_{L^{2}\left(0, t_{n} ; H^{m+1}(\Omega)\right)}+\left\|u\left(t_{n}\right)\right\|_{m+1}+\left\|v\left(t_{n}\right)\right\|_{m+1}\right)\right], \quad n=1,2, \ldots, N .
\end{aligned}
$$

Proof The result is proved by estimates (3.19) and (3.20) of the Theorem 2.

Theorem 3 Assume that the solution $u$ to Problem II is sufficiently smooth so that $u \in$ $H^{l+1}\left(0, T ; H^{1}(\Omega)\right) \cap H^{1}\left(0, T ; H^{m+1}(\Omega)\right), v_{t} \in L^{2}\left(0, T ; H^{m+1}(\Omega)\right)$, and $u(x, y, t) \in H^{m+1}(\Omega)$, $\forall t \in[0, T]$. Then we have the following error estimates:

$$
\begin{aligned}
\| u\left(t_{n}\right) & -u^{h k}\left(t_{n}\right) \|_{0} \\
\leq & C\left\{k^{l+1}\|\nabla u\|_{H^{l+1}\left(0, t_{n} ; L^{2}(\Omega)\right)}\right. \\
& \left.+h^{m+1}\left[\left\|u\left(t_{n}\right)\right\|_{m+1}+\left\|v_{t}\right\|_{L^{2}\left(0, t_{n} ; H^{m+1}(\Omega)\right)}\right]\right\}, \quad n=1,2, \ldots, N, \\
\|(u- & \left.u^{h k}\right)_{t} \|_{L^{2}\left(0, t_{n} ; L^{2}(\Omega)\right)} \\
\leq & C\left\{k^{l}\left[\|\nabla u\|_{H^{l+1}\left(0, t_{n} ; L^{2}(\Omega)\right)}+\|u\|_{H^{l+1}\left(0, t_{n} ; L^{2}(\Omega)\right)}\right]\right. \\
& \left.+h^{m+1}\left[\left\|u_{t}\right\|_{L^{2}\left(0, t_{n} ; H^{m+1}(\Omega)\right)}+\left\|v_{t}\right\|_{L^{2}\left(0, t_{n} ; H^{m+1}(\Omega)\right)}\right]\right\}, \quad n=1,2, \ldots, N .
\end{aligned}
$$


Proof Taking $(\phi, \varphi)=\left(T_{h}\left(P_{k} P_{h} u-u^{h k}\right), T_{h}\left(P_{h} v-v^{h k}\right)\right)$ in (3.15) and (3.16) and using the definition and symmetry property of $T_{h}$, we obtain

$$
\begin{aligned}
\int_{0}^{t_{n}} & {\left[\left(\left(P_{h} v-v^{h k}\right), T_{h}\left(P_{h} v-v^{h k}\right)_{t}\right)+\varepsilon\left(P_{h} v-v^{h k},\left(P_{k} P_{h} u-u^{h k}\right)_{t}\right)\right.} \\
& +\gamma\left(\left(P_{k} P_{h} u-u^{h k}\right),\left(P_{k} P_{h} u-u^{h k}\right)_{t}\right) \mathrm{d} t \\
= & \left.\int_{0}^{t_{n}}\left(\left(P_{h} v-v\right)_{t}, T_{h}\left(P_{k} P_{h} u-u^{h k}\right)_{t}\right)\right] \mathrm{d} t \\
& +\gamma \int_{0}^{t_{n}}\left[\left(\nabla\left(P_{k} u-u\right), \nabla T_{h}\left(P_{k} P_{h} u-u^{h k}\right)_{t}\right)\right] \mathrm{d} t .
\end{aligned}
$$

In addition, setting $\varphi=P_{k} P_{h} u-u^{h k}$ in (3.16), from (3.30) we have

$$
\begin{aligned}
& \int_{0}^{t_{n}}\left[\left(\left(P_{h} v-v^{h k}\right), T_{h}\left(P_{h} v-v^{h k}\right)_{t}\right)+\varepsilon\left(\left(P_{k} P_{h} u-u^{h k}\right)_{t},\left(P_{k} P_{h} u-u^{h k}\right)_{t}\right)\right. \\
& \left.\quad+\gamma\left(\left(P_{k} P_{h} u-u^{h k}\right),\left(P_{k} P_{h} u-u^{h k}\right)_{t}\right)\right] \mathrm{d} t \\
& =\int_{0}^{t_{n}}\left(\left(P_{h} v-v\right)_{t}, T_{h}\left(P_{k} P_{h} u-u^{h k}\right)_{t}\right) \mathrm{d} t \\
& \quad+\gamma \int_{0}^{t_{n}}\left(\Delta\left(P_{k} u-u\right), T_{h}\left(P_{k} P_{h} u-u^{h k}\right)_{t}\right) \mathrm{d} t .
\end{aligned}
$$

By (3.6) and the Hölder and Cauchy inequalities applied to the right-hand side of (3.31) we obtain

$$
\begin{aligned}
\frac{1}{2} \| & T_{h}^{1 / 2}\left(P_{h} v-v^{h k}\right)\left(t_{n}\right)\left\|_{0}^{2}+\varepsilon\right\|\left(P_{k} P_{h} u-u^{h k}\right)_{t}\left\|_{L^{2}\left(0, t_{n} ; L^{2}(\Omega)\right)}^{2}+\frac{\gamma}{2}\right\|\left(P_{k} P_{h} u-u^{h k}\right)\left(t_{n}\right) \|_{0}^{2} \\
\leq & \frac{c}{\varepsilon}\left\|\left(P_{h} v-v\right)_{t}\right\|_{L^{2}\left(0, t_{n} ; L^{2}(\Omega)\right)}+\frac{\varepsilon}{4}\left\|\left(P_{k} P_{h} u-u^{h k}\right)_{t}\right\|_{L^{2}\left(0, t_{n} ; L^{2}(\Omega)\right)} \\
& +\frac{c \gamma^{2}}{\varepsilon}\left\|\nabla\left(P_{k} u-u\right)\right\|_{L^{2}\left(0, t_{n} ; L^{2}(\Omega)\right)}+\frac{\varepsilon}{4}\left\|\left(P_{k} P_{h} u-u^{h k}\right)_{t}\right\|_{L^{2}\left(0, t_{n} ; L^{2}(\Omega)\right)}
\end{aligned}
$$

Further, we have that

$$
\begin{aligned}
& \left\|T_{h}^{1 / 2}\left(P_{h} v-v^{h k}\right)\left(t_{n}\right)\right\|_{0}+\varepsilon\left\|\left(P_{k} P_{h} u-u^{h k}\right)_{t}\right\|_{L^{2}\left(0, t_{n} ; L^{2}(\Omega)\right)}+\gamma\left\|P_{k} P_{h} u\left(t_{n}\right)-u^{h k}\left(t_{n}\right)\right\|_{0} \\
& \quad \leq \frac{2 c}{\varepsilon}\left\|\left(P_{h} v-v\right)_{t}\right\|_{L^{2}\left(0, t_{n} ; L^{2}(\Omega)\right)}+\frac{2 c \gamma^{2}}{\varepsilon}\left\|\nabla\left(P_{k} u-u\right)\right\|_{L^{2}\left(0, t_{n} ; L^{2}(\Omega)\right)}
\end{aligned}
$$

Hence, employing the triangle inequality to (3.33), we obtain

$$
\begin{aligned}
\left\|u\left(t_{n}\right)-u^{h k}\left(t_{n}\right)\right\|_{0} \leq & \left\|u\left(t_{n}\right)-P_{h} P_{k} u\left(t_{n}\right)\right\|_{0}+\left\|P_{k} P_{h} u\left(t_{n}\right)-u^{h k}\left(t_{n}\right)\right\|_{0} \\
\leq & \left\|u\left(t_{n}\right)-P_{h} P_{k} u\left(t_{n}\right)\right\|_{0}+\frac{2 c}{\varepsilon \gamma}\left\|\left(P_{h} v-v\right)_{t}\right\|_{L^{2}\left(0, t_{n} ; L^{2}(\Omega)\right)} \\
& +\frac{2 c \gamma}{\varepsilon}\left\|\nabla\left(P_{k} u-u\right)\right\|_{L^{2}\left(0, t_{n} ; L^{2}(\Omega)\right)}
\end{aligned}
$$


and

$$
\begin{aligned}
\|(u- & \left.u^{h k}\right)_{t} \|_{L^{2}\left(0, t_{n} ; L^{2}(\Omega)\right)} \\
\leq & \left\|\left(u-P_{h} P_{k} u\right)_{t}\right\|_{L^{2}\left(0, t_{n} ; L^{2}(\Omega)\right)}+\left\|\left(P_{k} P_{h} u-u^{h k}\right)_{t}\right\|_{L^{2}\left(0, t_{n} ; L^{2}(\Omega)\right)} \\
\leq & \left\|\left(u-P_{h} P_{k} u\right)_{t}\right\|_{L^{2}\left(0, t_{n} ; L^{2}(\Omega)\right)}+\frac{2 c}{\varepsilon^{2}}\left\|\left(P_{h} v-v\right)_{t}\right\|_{L^{2}\left(0, t_{n} ; L^{2}(\Omega)\right)} \\
& +\frac{2 c \gamma^{2}}{\varepsilon^{2}}\left\|\nabla\left(P_{k} u-u\right)\right\|_{L^{2}\left(0, t_{n} ; L^{2}(\Omega)\right)} .
\end{aligned}
$$

Theorem 3 now follows from (3.34), (3.35), and Lemma 1.

Theorem 4 Under the assumptions of Theorem 3, we have the following estimate:

$$
\begin{aligned}
\left\|u(t)-u^{h k}(t)\right\|_{L^{2}\left(L^{2}\right)} \leq & C\left\{k^{l+1}\left[\|u\|_{H^{l+1}\left(L^{2}\right)}+\|\nabla u\|_{H^{l+1}\left(L^{2}\right)}\right]\right. \\
& \left.+h^{m+1}\left[\sup _{0 \leq t \leq T}\|u\|_{m+1}+\left\|u_{t}\right\|_{L^{2}\left(H^{m+1}\right)}+\left\|v_{t}\right\|_{L^{2}\left(H^{m+1}\right)}\right]\right\} .
\end{aligned}
$$

Proof Let $t \in[0, T]$ belonging to some interval $t \in\left[t_{n-1}, t_{n}\right]$, we have the following identity:

$$
u(t)-u^{h k}(t)=\int_{t_{n-1}}^{t}\left(u-u^{h k}\right)_{t} \mathrm{~d} t+u\left(t_{n-1}\right)-u^{h k}\left(t_{n-1}\right)
$$

It follows from (3.37) and the Hölder inequality that

$$
\begin{aligned}
\left\|u(t)-u^{h k}(t)\right\|_{0} & \leq \int_{t_{n-1}}^{t_{n}}\left\|\left(u-u^{h k}\right)_{t}\right\|_{0} \mathrm{~d} t+\left\|u\left(t_{n-1}\right)-u^{h k}\left(t_{n-1}\right)\right\|_{0} \\
& \leq k^{\frac{1}{2}}\left(\int_{t_{n-1}}^{t_{n}}\left\|\left(u-u^{h k}\right)_{t}\right\|_{0}^{2} \mathrm{~d} t\right)^{\frac{1}{2}}+\left\|u\left(t_{n-1}\right)-u^{h k}\left(t_{n-1}\right)\right\|_{0} .
\end{aligned}
$$

Further, squaring both sides of (3.38) and then integrating with respect to $t$ from $t_{n-1}$ to $t_{n}$, we have

$$
\begin{aligned}
\int_{t_{n-1}}^{t_{n}}\left\|u(t)-u^{h k}(t)\right\|_{0}^{2} \mathrm{~d} t \leq & c\left(k \int_{t_{n-1}}^{t_{n}}\left\|\left(u-u^{h k}\right)_{t}\right\|_{L^{2}\left(t_{n-1}, t_{n} ; L^{2}(\Omega)\right)}^{2} \mathrm{~d} t\right. \\
& \left.+\int_{t_{n-1}}^{t_{n}}\left\|u\left(t_{n-1}\right)-u^{h k}\left(t_{n-1}\right)\right\|_{0}^{2} \mathrm{~d} t\right) .
\end{aligned}
$$

By summing (3.39) from 1 to $N$ we obtain

$$
\begin{aligned}
\left\|u(t)-u^{h k}(t)\right\|_{L^{2}\left(0, T ; L^{2}(\Omega)\right)}^{2} \leq & c\left(k^{2}\left\|\left(u-u^{h k}\right)_{t}\right\|_{L^{2}\left(0, T ; L^{2}(\Omega)\right)}^{2}\right. \\
& \left.+\int_{0}^{T}\left\|u\left(t_{n-1}\right)-u^{h k}\left(t_{n-1}\right)\right\|_{0}^{2} \mathrm{~d} t\right) .
\end{aligned}
$$

Finally, (3.36) directly follows from (3.40) and Theorem 3. 


\section{Numerical experiments}

In this section, we provide two numerical examples to verify the efficiency and feasibility of the STCFE algorithm. Moreover, we demonstrate that the numerical results are consistent with theoretical ones. We investigate problem I on the unit spatial region $\Omega=[0,1] \times$ $[0,1]$ and temporal interval $[0,1]$. Let $u_{N}=u\left(t_{N}\right)$. We take linear polynomials of spatial variables and quadratic polynomials of temporal variable, that is, $m=1$ and $l=2$. All the experiments are implemented on unstructured meshes, just as the partition presented in Figure 1 with $h=1 / 16$, and computations were performed from $t=0$ to the final time $T=1$. In addition, we also give the errors and convergence rates in the $H^{1}$ norm of $v$ at $t=t_{N}$ and in the $L^{2}\left(H^{1}\right)$ norm of $u$.

In the first example, we take $\varepsilon=\gamma=1$. The exact solution $u=e^{-t} \sin (2 \pi x) \sin (2 \pi y), v=$ $-e^{-t} \sin (2 \pi x) \sin (2 \pi y)$ is determined by (2.1) if $f=-e^{-t} \sin (2 \pi x) \sin (2 \pi y), u_{0}=\sin (2 \pi x) \times$ $\sin (2 \pi y)$, and $v_{0}=-\sin (2 \pi x) \sin (2 \pi y)$. First, we study the rates of convergence in spatial variables. To this end, we consider our STCFE discretization on a sequence of successive refinements of spatial grids with fixed time step $k=0.001$. Table 1 , Table 3 , and Table 5 show the errors and the rates of convergence of $u$ in the $L^{2}, H^{1}, L^{2}\left(L^{2}\right)$, and $L^{2}\left(H^{1}\right)$ norms and of $v$ in the $L^{2}$ and $H^{1}$ norms with respect to spatial variables, respectively. From these tables we can see that the second-order accuracy in space in the $L^{2}$ and $L^{2}\left(L^{2}\right)$ norms and the first-order accuracy in space in the $H^{1}$ and $L^{2}\left(H^{1}\right)$ norms are derived, respectively, which are consistent with theoretical results. Furthermore, the plots of numerical and exact solutions with $h=1 / 32$ in Figures 2 and 3 for $u$ and in Figures 4 and 5 for $v$ are provided, respectively. From these figures we can see that the numerical solutions approximate the exact ones very well.

Figure 1 A partition of domain with $h=1 / 16$.

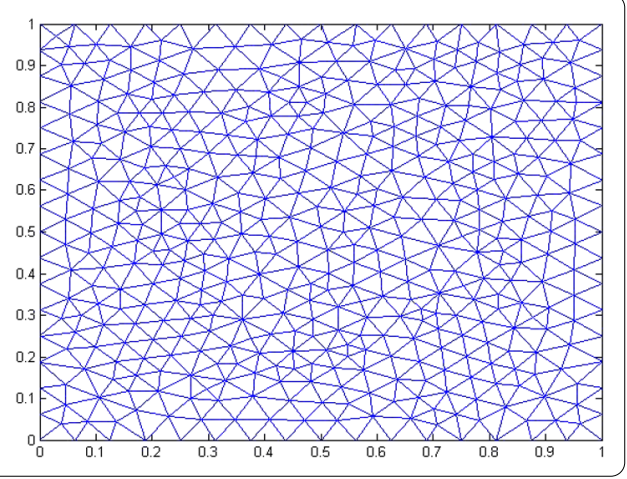

Figure 2 The numerical solution of $u$ at $t=1$.

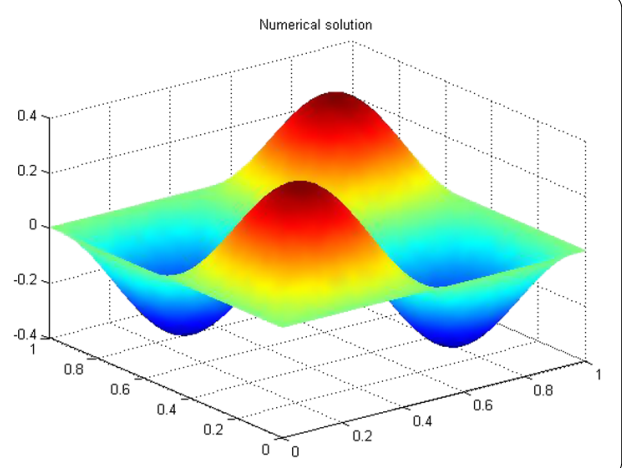




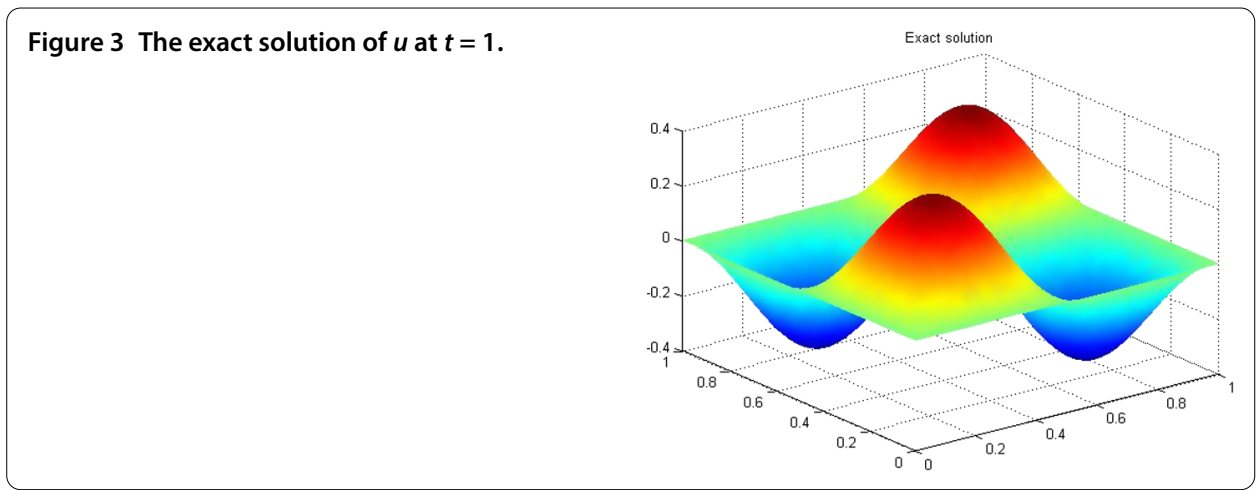

Figure 4 The numerical solution of $v$ at $t=1$.

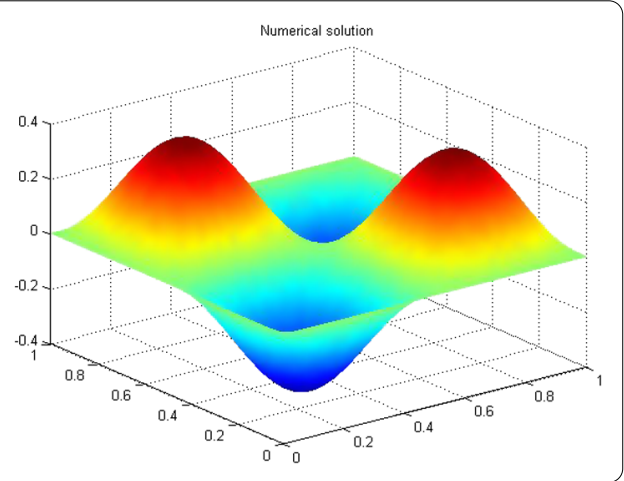

Figure 5 The exact solution of $v$ at $t=1$.
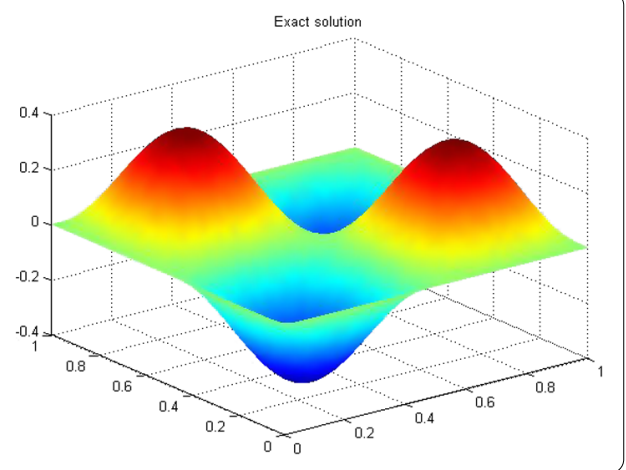

Now, we study the convergence rates with respect to temporal variable. Since we take $l=2$, the optimal convergence rates in the $L^{2}, L^{2}\left(L^{2}\right), H^{1}$, and $L^{2}\left(H^{1}\right)$ norms should theoretically be of the third-order accuracy in time. Therefore, in order to test the convergence rates in temporal variable, we take $h=O\left(k^{3 / 2}\right)$ and $h=O\left(k^{3}\right)$, respectively, so that the errors in the $L^{2}, L^{2}\left(L^{2}\right), H^{1}$, and $L^{2}\left(H^{1}\right)$ norms would be optimal in time. Table 2 , Table 4 , and Table 6 indicate that the rates of convergence of $u$ and $v$ in time are close to the third-order accuracy, which is also consistent with theoretical results. Here, the rates of convergence with respect to time are calculated by the formula

$$
\text { Rate }=\frac{\log \left(e_{2} / e_{1}\right)}{\log \left(k_{2} / k_{1}\right)},
$$

where $k_{1}, k_{2}$ and $e_{1}, e_{2}$ are successive time steps and errors, respectively. 
Table 1 The errors and convergence rates of $u$ at $t=t_{N}$ in the $L^{2}$ and $H^{1}$ norms in space

\begin{tabular}{lllll}
\hline $\boldsymbol{h}$ & $\boldsymbol{\| \boldsymbol { u } _ { \boldsymbol { N } } - \boldsymbol { u } _ { \boldsymbol { N } } ^ { \boldsymbol { h } } \boldsymbol { \| } _ { \boldsymbol { L } \mathbf { 2 } }}$ & Rate & $\left\|\boldsymbol{u}_{\boldsymbol{N}}-\boldsymbol{u}_{\boldsymbol{N}}^{\boldsymbol{h}}\right\|_{\boldsymbol{H}} \mathbf{1}$ & Rate \\
\hline $1 / 8$ & $1.4439 \mathrm{e}-2$ & & $4.8570 \mathrm{e}-1$ & \\
$1 / 16$ & $3.5585 \mathrm{e}-3$ & 2.0207 & $2.4646 \mathrm{e}-1$ & 0.9787 \\
$1 / 32$ & $9.0532 \mathrm{e}-4$ & 1.9748 & $1.2406 \mathrm{e}-1$ & 0.9903 \\
$1 / 64$ & $2.3248 \mathrm{e}-4$ & 1.9613 & $6.1777 \mathrm{e}-2$ & 1.0059 \\
\hline
\end{tabular}

Table 2 The errors and convergence rates of $u$ at $t=t_{N}$ in the $L^{2}$ and $H^{1}$ norms in time

\begin{tabular}{llllll}
\hline$(\boldsymbol{h}, \boldsymbol{k})$ & $\boldsymbol{\|} \boldsymbol{u}_{\boldsymbol{N}}-\boldsymbol{u}_{\boldsymbol{N}}^{\boldsymbol{h} \boldsymbol{k}} \|_{\boldsymbol{L}^{\mathbf{2}}}$ & Rate & $(\boldsymbol{h}, \boldsymbol{k})$ & $\left\|\boldsymbol{u}_{\boldsymbol{N}}-\boldsymbol{u}_{\boldsymbol{N}}^{\boldsymbol{h k}}\right\|_{\boldsymbol{H}^{\mathbf{1}}}$ & Rate \\
\hline$(0.125,0.25)$ & $7.1268 \mathrm{e}-3$ & & $(1 / 8,1 / 2)$ & $2.0646 \mathrm{e}-1$ & \\
$(0.0442,0.125)$ & $8.3093 \mathrm{e}-4$ & 3.1005 & $(1 / 27,1 / 3)$ & $6.0569 \mathrm{e}-2$ & 3.0244 \\
$(0.0156,0.0625)$ & $1.0239 \mathrm{e}-4$ & 3.0206 & $(1 / 64,1 / 4)$ & $2.5331 \mathrm{e}-2$ & 3.0302 \\
\hline
\end{tabular}

Table 3 The errors and convergence rates of $v$ at $t=t_{N}$ in $L^{2}$ and $H^{1}$ norms about space

\begin{tabular}{|c|c|c|c|c|}
\hline $\boldsymbol{h}$ & $\left\|v_{N}-v_{N}^{h k}\right\|_{L^{2}}$ & Rate & $\left\|v_{N}-v_{N}^{h k}\right\|_{H^{1}}$ & Rate \\
\hline $1 / 8$ & $1.4566 \mathrm{e}-2$ & & $4.8570 \mathrm{e}-1$ & \\
\hline $1 / 16$ & $3.5932 \mathrm{e}-3$ & 2.0192 & $2.4646 \mathrm{e}-1$ & 0.9787 \\
\hline $1 / 32$ & $9.1396 \mathrm{e}-3$ & 1.9751 & $1.2406 \mathrm{e}-1$ & 0.9903 \\
\hline $1 / 64$ & $2.3479 \mathrm{e}-4$ & 1.9608 & $6.1777 \mathrm{e}-2$ & 1.0059 \\
\hline
\end{tabular}

Table 4 The errors and convergence rates of $v$ at $t=t_{N}$ in $L^{2}$ and $H^{1}$ norms about time

\begin{tabular}{llllll}
\hline$(\boldsymbol{h}, \boldsymbol{k})$ & $\boldsymbol{\|} \boldsymbol{v}_{\boldsymbol{N}}-\boldsymbol{v}_{\boldsymbol{N}}^{\boldsymbol{h} \boldsymbol{k}} \boldsymbol{\|}_{\boldsymbol{L}} \mathbf{2}$ & Rate & $(\boldsymbol{h}, \boldsymbol{k})$ & $\| \boldsymbol{v}_{\boldsymbol{N}}-\boldsymbol{v}_{\boldsymbol{N}}^{\boldsymbol{h} \boldsymbol{k}} \boldsymbol{H}_{\boldsymbol{H}}$ & Rate \\
\hline$(0.125,1 / 4)$ & $7.1701 \mathrm{e}-3$ & & $(1 / 8,1 / 2)$ & $2.0643 \mathrm{e}-1$ & \\
$(0.0442,1 / 8)$ & $8.3938 \mathrm{e}-4$ & 3.0946 & $(1 / 27,1 / 3)$ & $6.0569 \mathrm{e}-2$ & 3.0241 \\
$(0.0156,1 / 16)$ & $1.0343 \mathrm{e}-4$ & 3.0202 & $(1 / 64,1 / 4)$ & $2.5331 \mathrm{e}-2$ & 3.0302 \\
\hline
\end{tabular}

Table 5 The errors and convergence rates of $u$ in $L^{2}\left(L^{2}\right)$ and $L^{2}\left(H^{1}\right)$ norms about space

\begin{tabular}{lllll}
\hline $\boldsymbol{h}$ & $\boldsymbol{\|} \boldsymbol{u}-\boldsymbol{u}^{\boldsymbol{h} \boldsymbol{k}} \|_{\boldsymbol{L}^{\mathbf{2}}\left(\mathbf{L}^{\mathbf{2}}\right)}$ & Rate & $\left\|\boldsymbol{u}-\boldsymbol{u}^{\boldsymbol{h k}}\right\|_{\boldsymbol{L}^{\mathbf{2}}\left(\boldsymbol{H}^{\mathbf{1}}\right)}$ & Rate \\
\hline $1 / 8$ & $1.0458 \mathrm{e}-2$ & & $3.5296 \mathrm{e}-1$ & \\
$1 / 16$ & $2.5742 \mathrm{e}-3$ & 2.0224 & $1.7909 \mathrm{e}-1$ & 0.9788 \\
$1 / 32$ & $6.5257 \mathrm{e}-4$ & 1.9799 & $9.0149 \mathrm{e}-2$ & 0.9903 \\
$1 / 64$ & $1.6514 \mathrm{e}-4$ & 1.9824 & $4.4891 \mathrm{e}-2$ & 1.0059 \\
\hline
\end{tabular}

Table 6 The errors and convergence rates of $u$ in the $L^{2}\left(L^{2}\right)$ and $L^{2}\left(H^{1}\right)$ norms in time

\begin{tabular}{|c|c|c|c|c|c|}
\hline$(h, k)$ & $\left\|u-u^{h k}\right\|_{L^{2}\left(L^{2}\right)}$ & Rate & $(h, k)$ & $\left\|u-u^{h k}\right\|_{L^{2}\left(H^{1}\right)}$ & Rate \\
\hline$(0.125,1 / 4)$ & $1.0053 e-2$ & & $(1 / 8,1 / 2)$ & $3.6314 \mathrm{e}-1$ & \\
\hline$(0.0442,1 / 8)$ & $1.3166 \mathrm{e}-3$ & 2.9327 & $(1 / 27,1 / 3)$ & $1.0685 \mathrm{e}-1$ & 3.0171 \\
\hline$(0.0156,1 / 16)$ & $1.6673 \mathrm{e}-4$ & 2.9817 & $(1 / 64,1 / 4)$ & $4.4870 e-2$ & 3.0161 \\
\hline
\end{tabular}

In our second example, we take $\varepsilon=\gamma=0.001$. The exact solution $u=e^{-t} \sin (2 \pi x) \times$ $\sin (2 \pi y), v=-e^{-t} \sin (2 \pi x) \sin (2 \pi y)$ is also determined by (2.1) if $f=-e^{-t} \sin (2 \pi x) \sin (2 \pi y)$, $u_{0}=\sin (2 \pi x) \sin (2 \pi y)$, and $v_{0}=-\sin (2 \pi x) \sin (2 \pi y)$. In the same way of studying convergence rates as in the first example, we list the errors and rates of convergence in Tables 7 12. From these tables we know that the second-order accuracy in the $L^{2}$ and $L^{2}\left(L^{2}\right)$ norms and the first-order accuracy in the $H^{1}$ and $L^{2}\left(H^{1}\right)$ norms with respect to space and the third-order accuracy in the $L^{2}, H^{1}, L^{2}\left(L^{2}\right)$, and $L^{2}\left(H^{1}\right)$ norms with respect to time are also derived, which further verify the efficiency and feasibility of the STCFE method. 
Table 7 The errors and convergence rates of $u$ at $t=t_{N}$ in the $L^{2}$ and $H^{1}$ norms in space

\begin{tabular}{lllll}
\hline $\boldsymbol{h}$ & $\boldsymbol{\| \boldsymbol { u } _ { \boldsymbol { N } } - \boldsymbol { u } _ { \boldsymbol { N } } ^ { \boldsymbol { h } } \| _ { \boldsymbol { L } ^ { \mathbf { 2 } } }}$ & Rate & $\left\|\boldsymbol{u}_{\boldsymbol{N}}-\boldsymbol{u}_{\boldsymbol{N}}^{\boldsymbol{h}}\right\|_{\boldsymbol{H}} \mathbf{1}$ & Rate \\
\hline $1 / 8$ & $2.9254 \mathrm{e}-3$ & & $2.0047 \mathrm{e}-1$ & \\
$1 / 16$ & $6.8669 \mathrm{e}-4$ & 2.0909 & $1.0049 \mathrm{e}-1$ & 0.9963 \\
$1 / 32$ & $1.8210 \mathrm{e}-4$ & 1.9149 & $5.0419 \mathrm{e}-2$ & 0.9950 \\
$1 / 64$ & $4.5634 \mathrm{e}-5$ & 1.9965 & $2.5113 \mathrm{e}-2$ & 1.0055 \\
\hline
\end{tabular}

Table 8 The errors and convergence rates of $u$ at $t=t_{N}$ in the $L^{2}$ and $H^{1}$ norms in time

\begin{tabular}{llllll}
\hline $\boldsymbol{( h , \boldsymbol { k } )}$ & $\boldsymbol{\| \boldsymbol { u } _ { \boldsymbol { N } } - \boldsymbol { u } _ { \boldsymbol { N } } ^ { \boldsymbol { h } \boldsymbol { k } } \boldsymbol { L } _ { \boldsymbol { L } } \mathbf { 2 }}$ & Rate & $(\boldsymbol{h}, \boldsymbol{k})$ & $\left\|\boldsymbol{u}_{\boldsymbol{N}}-\boldsymbol{u}_{\boldsymbol{N}}^{\boldsymbol{h k}}\right\|_{\boldsymbol{H}^{\mathbf{1}}}$ & Rate \\
\hline$(0.125,0.25)$ & $4.6260 \mathrm{e}-3$ & & $(1 / 8,1 / 2)$ & $2.0234 \mathrm{e}-1$ & \\
$(0.0442,0.125)$ & $4.3551 \mathrm{e}-4$ & 3.4090 & $(1 / 27,1 / 3)$ & $6.0357 \mathrm{e}-2$ & 2.9834 \\
$(0.0156,0.0625)$ & $5.0465 \mathrm{e}-5$ & 3.1093 & $(1 / 64,1 / 4)$ & $2.5314 \mathrm{e}-2$ & 3.0204 \\
\hline
\end{tabular}

Table 9 The errors and convergence rates of $v$ at $t=t_{N}$ in the $L^{2}$ and $H^{1}$ norms in space

\begin{tabular}{lllll}
\hline $\boldsymbol{h}$ & $\boldsymbol{\| \boldsymbol { v } _ { \boldsymbol { N } } - \boldsymbol { v } _ { \boldsymbol { N } } ^ { \boldsymbol { h } \boldsymbol { k } } \boldsymbol { \| } _ { \boldsymbol { L } \mathbf { 2 } }}$ & Rate & $\| \boldsymbol{v}_{\boldsymbol{N}}-\boldsymbol{v}_{\boldsymbol{N}}^{\boldsymbol{h} \boldsymbol{k}} \boldsymbol{H}_{\boldsymbol{H}} \mathbf{1}$ & Rate \\
\hline $1 / 8$ & $1.3422 \mathrm{e}-2$ & & $2.1612 \mathrm{e}-1$ & \\
$1 / 16$ & $3.3845 \mathrm{e}-3$ & 1.9876 & $1.0199 \mathrm{e}-1$ & 1.0833 \\
$1 / 32$ & $8.5281 \mathrm{e}-4$ & 1.9887 & $5.0673 \mathrm{e}-2$ & 1.0092 \\
$1 / 64$ & $2.2460 \mathrm{e}-4$ & 1.9249 & $2.5147 \mathrm{e}-2$ & 1.0108 \\
\hline
\end{tabular}

Table 10 The errors and convergence rates of $v$ at $t=t_{N}$ in the $L^{2}$ and $H^{1}$ norms in time

\begin{tabular}{llllll}
\hline$(\boldsymbol{h}, \boldsymbol{k})$ & $\boldsymbol{\|} \boldsymbol{v}_{\boldsymbol{N}}-\boldsymbol{v}_{\boldsymbol{N}}^{\boldsymbol{h} \boldsymbol{k}} \boldsymbol{\|}_{\boldsymbol{L}} \mathbf{2}$ & Rate & $(\boldsymbol{h}, \boldsymbol{k})$ & $\left\|\boldsymbol{v}_{\boldsymbol{N}}-\boldsymbol{v}_{\boldsymbol{N}}^{\boldsymbol{h} \boldsymbol{k}}\right\|_{\boldsymbol{H}^{\mathbf{1}}}$ & Rate \\
\hline$(0.125,1 / 4)$ & $1.3732 \mathrm{e}-2$ & & $(1 / 8,1 / 2)$ & $2.2904 \mathrm{e}-1$ & \\
$(0.0442,1 / 8)$ & $1.7806 \mathrm{e}-3$ & 2.9471 & $(1 / 27,1 / 3)$ & $6.1171 \mathrm{e}-2$ & 3.2561 \\
$(0.0156,1 / 16)$ & $2.2889 \mathrm{e}-4$ & 2.9596 & $(1 / 64,1 / 4)$ & $2.5366 \mathrm{e}-2$ & 3.0598 \\
\hline
\end{tabular}

Table 11 The errors and convergence rates of $u$ in the $L^{2}\left(L^{2}\right)$ and $L^{2}\left(H^{1}\right)$ norms in space

\begin{tabular}{lllll}
\hline $\boldsymbol{h}$ & $\boldsymbol{\|} \boldsymbol{u}-\boldsymbol{u}^{\boldsymbol{h} \boldsymbol{k}} \|_{\mathbf{L}^{\mathbf{2}}\left(\mathbf{L}^{\mathbf{2}}\right)}$ & Rate & $\left\|\boldsymbol{u}-\boldsymbol{u}^{\boldsymbol{h} \boldsymbol{k}}\right\|_{\mathbf{L}^{\mathbf{2}}\left(\boldsymbol{H}^{\mathbf{1}}\right)}$ & Rate \\
\hline $1 / 8$ & $9.5581 \mathrm{e}-3$ & & $3.5267 \mathrm{e}-1$ & \\
$1 / 16$ & $2.3449 \mathrm{e}-3$ & 2.0272 & $1.7901 \mathrm{e}-1$ & 0.9782 \\
$1 / 32$ & $5.9563 \mathrm{e}-4$ & 1.9771 & $9.0108 \mathrm{e}-2$ & 0.9903 \\
$1 / 64$ & $1.5021 \mathrm{e}-4$ & 1.9874 & $4.4884 \mathrm{e}-2$ & 1.0054 \\
\hline
\end{tabular}

Table 12 The errors and convergence rates of $u$ in the $L^{2}\left(L^{2}\right)$ and $L^{2}\left(H^{1}\right)$ norms in time

\begin{tabular}{llllll}
\hline $\boldsymbol{( h ,}, \boldsymbol{k})$ & $\boldsymbol{\| u}-\boldsymbol{u}^{\boldsymbol{h} \boldsymbol{k}} \boldsymbol{\|}_{\mathbf{L}^{\mathbf{2}}\left(\mathbf{L}^{\mathbf{2}}\right)}$ & Rate & $\mathbf{( h , k )}$ & $\| \boldsymbol{u}-\boldsymbol{u}^{\boldsymbol{h} \boldsymbol{k}} \boldsymbol{\|}_{\mathbf{L}^{\mathbf{2}}\left(\boldsymbol{H}^{\mathbf{1}}\right)}$ & Rate \\
\hline$(0.125,1 / 4)$ & $9.49444 \mathrm{e}-2$ & & $(1 / 8,1 / 2)$ & $3.6262 \mathrm{e}-1$ & \\
$(0.0442,1 / 8)$ & $1.2179 \mathrm{e}-3$ & 2.9627 & $(1 / 27,1 / 3)$ & $1.0680 \mathrm{e}-1$ & 3.0148 \\
$(0.0156,1 / 16)$ & $1.5288 \mathrm{e}-4$ & 2.9940 & $(1 / 64,1 / 4)$ & $4.4864 \mathrm{e}-2$ & 3.0149 \\
\hline
\end{tabular}

\section{Conclusions and perspectives}

In this article, we have developed the STCFE method for the 2D second-order viscoelastic wave equation. The existence, uniqueness, and stability of the STCFE solutions are demonstrated, and the optimal error estimates in the $L^{2}, H^{1}$, and $L^{2}\left(L^{2}\right)$ norms of $u$ and in the $L^{2}$ norm of $v$ are provided. However, our theoretical analysis is different from those in [15-17]; our method is more simple and convenient and easier for obtaining various error estimates in different norms, and so it has a more important meaning. In addition, our error estimates do not require any restriction conditions on the spatial and temporal grid sizes. Thus, the method used here is a kind of improvement and development for the 
existing works. Finally, the numerical examples illustrate that the numerical results are consistent with the theoretical ones and verify the efficiency and feasibility of the STCFE method.

In the future work, we intend to employ the ideas of this work to establish the STCFE models for more complex linear and nonlinear TDPDEs. Moreover, the STCFE method enhance the accuracy of numerical solutions, but they include many degrees of freedom; therefore, in the next work, we will aim to establish the reduced-order STCFE extrapolating algorithm based on proper orthogonal decomposition.

\section{Competing interests}

The authors declare that they have no competing interests.

\section{Authors' contributions}

All authors contributed equally and significantly in writing this article. All authors read and approved the final manuscript.

\section{Author details}

'School of Mathematical Sciences, Inner Mongolia University, No. 235, West Road of University, Saihan District, Hohhot, 010021, China. ${ }^{2}$ School of Mathematics and Physics, North China Electric Power University, No. 2, Bei Nong Road, Changping District, Beijing, 102206, China.

\section{Acknowledgements}

This research was supported by National Science Foundation of China grant $(11361035,11271127,11301258)$. Natural Science Foundation of Inner Mongolia (2014BS0101).

Received: 14 September 2015 Accepted: 15 February 2016 Published online: 25 February 2016

\section{References}

1. Gurtin, M, Pipkin, A: A general theory of heat conduction with finite wave speeds. Arch. Ration. Mech. Anal. 31 , 113-126 (1968)

2. Yuan, YR: Finite difference method and analysis for three-dimensional semiconductor device of heat conduction. Sci. China Ser. A 11, 21-32 (1996)

3. Yuan, YR, Wang, H: Error estimates for the finite element methods of nonlinear hyperbolic equations. J. Syst. Sci. Math. Sci. 5(3), 161-171 (1985)

4. Lin, YP: A mixed boundary problem describing the propagation of disturbances in viscous media solution for quasi-linear equations. J. Math. Anal. Appl. 135, 644-653 (1988)

5. Suveika, K: Mixed problems for an equation describing the propagation of disturbances in viscous media. J. Differ. Equ. 19, 337-347 (1982)

6. Raynal, M: On some nonlinear problems of diffusion in Volterra equations. In: London, S, Staffans, O (eds.) Lecture Notes in Mathematics, vol. 737, pp. 251-266. Springer, Berlin (1979)

7. Cannon, JR, Lin, Y: A priori $L^{2}$ error estimates for finite-element methods for nonlinear diffusion equations with memory. SIAM J. Numer. Anal. 27(3), 595-607 (1990)

8. Luo, ZD: The Foundations and Applications of Mixed Finite Element Methods. Chinese Science Press, Beijing (2006) (in Chinese)

9. Thomée, V: Galerkin Finite Element Methods for Parabolic Problems. Lecture Notes in Mathematics, vol. 1054. Springer, Berlin (1984)

10. Thomée, V: Negative norm estimates and superconvergence in Galerkin methods for parabolic problems. Math. Comput. 34, 93-113 (1980)

11. French, DA, Schaeffer, JW: Continuous finite element methods which preserve energy properties for nonlinear problems. Appl. Math. Comput. 39, 271-295 (1991)

12. Aziz, AK, Monk, P: Continuous finite elements in space and time for the heat equation. Math. Comput. 52, 255-274 (1989)

13. Karakashia, O, Makkridakis, C: A time-space finite element method for nonlinear Schrödinger equation: the continuous Galerkin method. Math. Comput. 97(222), 479-499 (1998)

14. Li, H, Liu, RX: The space-time finite element method for parabolic problems. Appl. Math. Mech. 22(6), 687-700 (2001)

15. Aziz, AK, Monk, P: Continuous finite elements in space and time for the heat equation. Math. Comput. 52, 255-274 (1989)

16. Bales, L, Lasiecka, I: Continuous finite elements in space and time for the nonhomogeneous wave equation. Comput. Math. Appl. 27(3), 91-102 (1994)

17. French, DA, Peterson, TE: A continuous space-time finite element method for the wave equation. Math. Comput. 65(214), 491-506 (1996)

18. Liu, Y, Li, H, He, S: Mixed time discontinuous space-time finite element method for convection diffusion equations. Appl. Math. Mech. 29(12), 1579-1586 (2008)

19. French, DA: A space-time finite element method for the wave equation. Comput. Methods Appl. Mech. Eng. 107, 145-157 (1993)

20. Adams, RA: Sobolev Spaces. Academic Press, New York (1975)

21. Brezzi, F, Fortin, M: Mixed and Hybrid Finite Element Methods. Springer, New York (1991)

22. Ciarlet, PG: The Finite Element Method for Elliptic Problems. North-Holland, Amsterdam (1978) 